As from January 1985 the British Journal of Industrial Medicine will appear monthly. This will ensure that papers can be published more quickly than has been possible during the past two or three years and also allow new features to be introduced. The new journal will publish original papers of a high standard as before and short reports and case reports. The information section will be retained and in addition editorials and letters to the Editor will be published. With the enlarged format it will also be possible to include more book reviews and notices.

Guidance for authors is given in detail below and will be published annually. All those who submit material to the journal are urged to follow the instructions carefully since failure to do so will inevitably cause delays in the consideration and publication of their manuscripts.

\section{Guidance for authors}

The British Journal of Industrial Medicine publishes material which is relevant to any aspect of occupational health. Papers that deal with environmental medicine will also be considered. The journal publishes four types of communication, original papers, short reports (which may include reports of clinical material), editorials, and letters to the Editor. Reviews are normally specially commissioned, and authors should not submit a review without prior consultation with the Editor.

All material submitted to the journal must be typewritten on one side of the paper only with double spacing and wide margins. Manuscripts must be submitted in triplicate to the Editor, TUC Centenary Institute of Occupational Health, London School of Hygiene and Tropical Medicine, Keppel Street (Gower Street), London WC1E 7HT; they must conform with the recommendations given below. Manuscripts must be written in English and spelling must follow the conventions in the Oxford English Dictionary. Both SI units and their equivalents must be given throughout. Authors should note that clarity and brevity are virtues that are given great weight when considering a paper for publica. tion. The Editor cannot enter into correspondence about papers that are rejected as being unsuitable for publication and his decision is final.

Papers are accepted on the understanding that the work described has not appeared in whole elsewhere and that they are subject to editorial revision. Where the findings have been published elsewhere in part this must be clearly stated and the submitted manuscript should be accompanied by a copy of the publication that contains those findings. If part of the findings are contained in a manuscript which is under consideration elsewhere a copy of that manuscript should be included with that submitted to the journal. A letter giving consent to publication must be signed by all those whose name appears on the manuscript. Papers that describe studies carried out on human subjects must give evidence that the protocol was approved by an ethical committee and that all the subjects gave their informed consent.

There is no prescribed length for original papers but authors should not submit papers which exceed 10000 words (about 30 typewritten sheets) without first consulting the Editor.

Original papers should follow the requirements of the International Steering Committee of Medical Editors; details, with which authors should familiarise themselves, are to be found in the British Medical Journal (1979;i:532-5). Papers should be prefaced by an abstract of the argument and findings and should be more comprehensive than a summary. The abstract must not contain references. Short reports or case reports do not require an abstract.

Photographs on glossy paper should be submitted unmounted. Colour photographs will not be published unless the authors underwrite the cost of production. Charts and graphs should be carefully drawn in black ink on tracing linen, Bristol board, or stout white paper. Only one original of the figures need be submitted but two additional copies will be required. Legends to figures should be typed on a separate sheet of paper.

Tables should be kept to a minimum and should not contain material that is fully described in the text. They should be typed on separate sheets of paper. Long appendices should be avoided. When the author considers it essential to include large numbers of tables or long appendices it may be possible to print them at the end of the text in miniprint. The Editor should be consulted in advance.

Signed editorials will normally be specially commissioned and authors who are considering the submission of an editorial should do so only after consultation with the Editor. Letters to the Editor, which should not exceed 500 words, may deal with any topic and will be published as soon as space permits. When letters refer to papers that have appeared in the Journal, the authors of the original paper will be given the opportunity to reply. Unsolicited book reviews will not be published.

References The number of references in the text of papers submitted to the Journal should be kept to 
a minimum. They are required only if they are essential to the development of a hypothesis which the authors are proposing to test, if they are necessary for the development of an argument or discussion, or if they describe methods that are being used when the original account is too lengthy to be included in full. Exhaustive lists of references are frequently included in a paper more to impress than to instruct and should be avoided whenever possible; over-frequent reference to the authors' own papers is an indulgence best avoided.

All references in material submitted to the journal must conform with the so-called Vancouver style which is intended to standardise requirements for authors. The style is described in detail in the British Medical Journal (1979;i:532-5) and authors are urged to study it carefully. Manuscripts in which the references do not follow the prescribed style will be returned to be amended before they are considered for publication. The essentials of the style are as follows.

References should be numbered consecutively in the order in which they are first mentioned in the text by Arabic numerals above the line on each occasion the reference is cited. References cited only in tables or in legends to figures should be numbered in accordance with a sequence established by the first identification in the text of a particular table or illustration. The references must include: the names and initials of all authors (unless there are more than six, when only the first three should be given followed by et al); the title of journal articles or book chapters; the titles of journals abbreviated according to the style of the Index Medicus; the year and volume number; and the first and final page numbers of the article or chapter. Titles of books should be followed by place of publication, publisher, and year.

Examples of common forms of references are:

'International Steering Committee of Medical
Editors. Uniform requirements for manuscripts
submitted to biomedical journals. $\mathrm{Br}$ Med J
$1979 ; \mathrm{i}: 532-5$.
2 Soter NA, Wasserman SI, Austen KF. Cold urticaria:
release into the circulation of histamine and
eosinophil chemotactic factor of anaphylaxis during
cold challenge. $N$ Eng $J$ Med 1976;294:687-90.
${ }^{3}$ Weinstein L, Swartz MN. Pathogenic properties of
invading micro-organisms, In: Sodeman WA Jr,
Sodeman WA, eds. Pathologic physiology: mechan-
isms of disease. Philadelphia: W B Saunders,
1974:457-72.

Authors should note that references will not be checked or completed by the editorial office; responsibility for the accuracy and completeness of references lies solely with the author.

Proofs Contributors will receive one proof on which only minor alterations may be made. It is assumed that the submitted manuscript will have been carefully proofread and will be essentially correct.

Reprints Twenty five reprints will be supplied free of charge. Further copies will be available on the payment of a fee; the number of additional reprints required should be inserted on the form sent with the proofs. Reprints of letters to the Editor will not be available. 